\title{
Publisher Correction: Real options signaling game models for dynamic acquisition under information asymmetry
}

\author{
Chi Man Leung ${ }^{1}$ • Yue Kuen Kwok ${ }^{1}$
}

\section{Correction to: Decisions Econ Finan (2018) 41:35-63 https://doi.org/10.1007/s10203-018-0206-3}

In the original publication, the copyright holder was incorrectly published as 'SpringerVerlag Italia S.r.l., part of Springer Nature' instead of 'Associazione per la Matematica Applicata alle Scienze Economiche e Sociali (AMASES)'.

The original article can be found online at https://doi.org/10.1007/s10203-018-0206-3.

$\bowtie \quad$ Chi Man Leung

chimanleung@ust.hk

1 Department of Mathematics, Hong Kong University of Science and Technology, Hong Kong, China 